SU-ITP-92-12

March 1992

\title{
Hawking Radiation and Back-Reaction ${ }^{\star}$
}

\author{
LEONARD SussKind AND LÁRus ThORLACIUS \\ Department of Physics \\ Stanford University, Stanford, CA 94305
}

\begin{abstract}
The puzzles of black hole evaporation can be studied in the simplified context of $1+1$ dimensional gravity. The semi-classical equations of Callan, Giddings, Harvey and Strominger provide a consistent description of the evaporation process which we describe in detail. We consider the possibility that black hole evolution leads to massive stable remnants. We show that such zero temperature remnant solutions exist but we also prove that a decaying black hole cannot evolve into one of them. Finally we consider the issue of loss of quantum information behind the global event horizon which develops in these geometries. An analogy with a well known solvable system shows that there may be less to information than meets the eye.
\end{abstract}

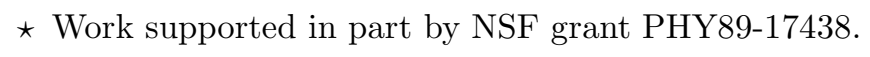




\section{Introduction}

Black hole evaporation provides us with an interesting set of puzzles where quantum mechanics and general relativity clash $[1,2,3]$. The central paradox is that matter in a quantum mechanically pure state can undergo gravitational collapse to form a black hole, which then evaporates, and in this process the initial pure state appears to evolve into a mixed quantum state, describing the outgoing Hawking radiation. This argument is based on a calculation which treats matter quantum mechanically in a background geometry which evolves according to Einstein's classical field equations. It seems prudent to include the perhaps subtle effects of back-reaction on the geometry, due to the emission of Hawking radiation, before we start tinkering with quantum mechanics. Hopefully these issues can be resolved without direct reference to Planck scale physics (after all the horizon of a massive black hole forms in a region of weak gravitational coupling) allowing us to explore interesting features of quantum gravity without having to grapple with a theory of everything.

It is often illuminating to address difficult physical problems in the simplified context of $1+1$ dimensions. A particularly interesting toy model for black hole physics was suggested by Callan, Giddings, Harvey and Strominger (CGHS) in reference [4], where they coupled two-dimensional gravity to a dilaton and conformal matter fields. CGHS described a set of exact classical solutions, including black holes formed by infalling shock waves, and they observed that Hawking radiation can be described by adding correction terms to the classical equations of motion. These extra terms derive from the conformal anomaly of the matter fields [5] and are local in conformal gauge, with the usual non-locality appearing only in the form of boundary conditions required to satisfy constraints. CGHS also pointed out that once these quantum corrections have been included one can study the back-reaction on the geometry, due to the Hawking radiation, using classical methods in the effective theory. They argued that the quantum corrections would prevent the usual black hole singularity from forming in gravitational collapse and therefore quantum information would not be lost. Subsequently it was shown that in this theory gravitational collapse always develops a singularity [6,7]. Thus the paradox has not been resolved.

In this paper we study the CGHS model further. Background material can be found in references [4], [6] and [7]. We begin in section 2 by briefly reviewing the model and describing some exact classical solutions. We then turn to the quantum theory in section 3. Following CGHS we study Hawking radiation and its back reaction on black holes using effective equations of motion, with correction terms induced by the conformal anomaly of the matter fields. Using both analytic and 
numerical methods we are able to present a fairly detailed picture of the formation and quantum mechanical evaporation of $1+1$ dimensional black holes.

An important issue to settle is the nature of the final state that a $1+1$ dimensional black hole will settle down to. One can think of a number of possibilities, all of which are problematic to some degree. The first, suggested by Hawking [2], is that the black hole completely evaporates leaving no remnant behind but only incoherent radiation, and quantum coherence is lost in the process. This is a radical proposal and more conservative alternatives should be exhausted before we modify quantum mechanics.

Another possibility is that the Hawking process leaves behind a stable remnant whose precise quantum state reflects the initial configuration of the system [8]. Evidently this requires a virtually infinite number of distinct quantum states of black hole cadavers. There are two very different cases to consider. On the one hand the mass and size of the remnants would be of order the Planck scale, in which case their density of states must be infinite. If these light remnant states couple to gravity in any conventional way, they would be copiously produced in pairs whenever there is available a Planck scale amount of energy. The energy would not have to be very concentrated because even if the probability for producing a particular pair of these Planck scale objects is tiny the infinite density of states makes the total rate diverge. For example, the sun would have been short lived indeed if such channels had been available. It has recently been suggested that the black hole remnants cannot be produced in pairs [6] but this in itself is a radical departure from the principles of relativistic quantum mechanics.

Alternatively the Hawking process might terminate while the black hole still has macroscopic mass. That could only happen if the back-reaction on the geometry develops a singularity on the apparent horizon itself. This may sound outlandish but in section 3 we will show that the curvature on the apparent horizon does in fact increase as the black hole evaporates and in section 4 we exhibit static, non-radiating solutions of the CGHS-equations which are candidates for such remnants. Massive remnants can carry a lot of information and their density of states would not have to diverge in order to preserve quantum coherence, so this possibility seems worth exploring. However, we are able to prove that it is not realized in $1+1$ dimensional gravitational collapse, as described by the CGHS-equations.

Perhaps the most conservative option is that despite its thermal appearance Hawking radiation really carries off information about the quantum state of the black hole [3]. This would reconcile the evolution of black holes with conventional quantum mechanics. In section 5 of this paper we will discuss to what extent this type of questions can be addressed in this $1+1$ dimensional model, but unfortunately we cannot offer any definitive conclusions at present. 


\section{The Model}

The system we will consider is two-dimensional gravity coupled to a dilaton $\phi$ and a set of scalar fields $f_{i}$. The classical dynamics is governed by the action

$$
S=\frac{1}{2 \pi} \int d^{2} x \sqrt{-g}\left[e^{-2 \phi}\left(R+4(\nabla \phi)^{2}+4 \lambda^{2}\right)-\frac{1}{2} \sum_{i=1}^{N}\left(\nabla f_{i}\right)^{2}\right],
$$

which resembles the target space action of $c=1$ non-critical string theory. It can be viewed as an effective action for radial modes of four-dimensional extreme dilaton black holes $[4,9,10]$ but in this paper we will study it on its own merits as a twodimensional model of gravity coupled to matter.

The matter fields $f_{i}$ are minimally coupled to the metric and do not directly interact with the dilaton. We will assume there is a large but finite number of them. The reason for this is that the one-loop quantum corrections to the action are dominated by the conformal anomaly of the matter fields if $N$ is large and the effective action takes a particularly simple form. The arbitrary parameter $\lambda$ defines a mass scale in this theory and we will refer to a black holes with $M>N \lambda$ as massive.

The classical equations of motion obtained from the action (2.1) are

$$
\begin{aligned}
\nabla^{2} f_{i} & =0 \\
R+4 \lambda^{2}+4 \nabla^{2} \phi-4(\nabla \phi)^{2} & =0 \\
2 e^{-2 \phi}\left[\nabla_{\mu} \nabla_{\nu} \phi+g_{\mu \nu}\left((\nabla \phi)^{2}-\nabla^{2} \phi-\lambda^{2}\right)\right] & =\frac{1}{2} \sum_{i=1}^{N}\left(\nabla_{\mu} f_{i} \nabla_{\nu} f_{i}-\frac{1}{2} g_{\mu \nu}\left(\nabla f_{i}\right)^{2}\right) .
\end{aligned}
$$

It is convenient to work in conformal gauge

$$
\begin{aligned}
& g_{+-}=g_{-+}=-\frac{1}{2} e^{2 \rho}, \\
& g_{--}=g_{++}=0,
\end{aligned}
$$

with light-cone coordinates $x^{ \pm}=x^{0} \pm x^{1}$. The fields in the theory are then $f_{i}, \phi$ and $\rho$, and the classical equations of motion become,

$$
\begin{aligned}
\partial_{+} \partial_{-} f_{i} & =0 \\
2 \partial_{+} \partial_{-} \phi-2 \partial_{+} \phi \partial_{-} \phi-\frac{\lambda^{2}}{2} e^{2 \rho} & =\partial_{+} \partial_{-} \rho, \\
\partial_{+} \partial_{-} \phi-2 \partial_{+} \phi \partial_{-} \phi-\frac{\lambda^{2}}{2} e^{2 \rho} & =0,
\end{aligned}
$$

respectively. In addition, one must impose as constraints the equations of motion 
corresponding to the metric components, which are set to zero in this gauge:

$$
\begin{aligned}
& e^{-2 \phi}\left(2 \partial_{+}^{2} \phi-4 \partial_{+} \rho \partial_{+} \phi\right)=\frac{1}{2} \sum_{i=1}^{N} \partial_{+} f_{i} \partial_{+} f_{i}, \\
& e^{-2 \phi}\left(2 \partial_{-}^{2} \phi-4 \partial_{-} \rho \partial_{-} \phi\right)=\frac{1}{2} \sum_{i=1}^{N} \partial_{-} f_{i} \partial_{-} f_{i} .
\end{aligned}
$$

A number of exact solutions of these equations can easily be found [4]. The simplest one is the vacuum solution

$$
\begin{aligned}
f_{i} & =0, \\
e^{-2 \phi}=e^{-2 \rho} & =-\lambda^{2} x^{+} x^{-} .
\end{aligned}
$$

This is just the 'linear dilaton' background of non-critical string theory. A change of variables, $x^{ \pm}= \pm e^{ \pm u^{ \pm}}$, makes the metric flat and the dilaton field linear in $u^{+}-u^{-}$. A 'static' black hole is described by

$$
\begin{aligned}
f_{i} & =0, \\
e^{-2 \phi}=e^{-2 \rho} & =\frac{M}{\lambda}-\lambda^{2} x^{+} x^{-} .
\end{aligned}
$$

Finally one can describe the metric and dilaton fields due to an infalling shell of matter by patching together the vacuum solution and a black hole solution across some light-like line $x^{+}=x_{0}^{+}$(see figure 1 ),

$$
e^{-2 \phi}=e^{-2 \rho}=\left\{\begin{array}{cc}
-\lambda^{2} x^{+} x^{-} & \text {if } x^{+}<x_{0}^{+} ; \\
-\frac{M}{\lambda x_{0}^{+}}\left(x^{+}-x_{0}^{+}\right)-\lambda^{2} x^{+} x^{-} & \text {if } x^{+}>x_{0}^{+} .
\end{array}\right.
$$

Note that $\phi$ and $\rho$ are continuous across the matching line. Inserting $\phi$ and $\rho$ from (2.8) into the ++ constraint equation in (2.5) reveals that this solution is indeed the response to an incoming shock wave with energy-momentum $\frac{1}{2} \sum_{i=1}^{N} \partial_{+} f_{i} \partial_{+} f_{i}=$ $\frac{M}{\lambda x_{0}^{+}} \delta\left(x^{+}-x_{0}^{+}\right)$. The geometry is singular on the curve where $e^{-2 \rho}=0$ and the line $x^{-}=-\frac{M}{\lambda^{3} x_{0}^{+}}$is a global event horizon in this classical solution.

The factor of $e^{-2 \phi}$ in front of the gravity terms in the action (2.1) makes the strength of gravitational quantum corrections depend on the magnitude of the dilaton field, $g \sim e^{\phi}$. Both the linear dilaton vacuum and the classical black hole solutions have a position dependent coupling strength with weak coupling being asymptotically far away. In fact, the strength of quantum corrections provides a 
coordinate invariant notion of position in this one-dimensional world. Recall that in the four-dimensional Schwarzschild solution lines of constant radial distance (and fixed angle) go from being time-like outside the horizon to being space-like inside the black hole. The analogous statement for our $1+1$ dimensional black holes is that $(\nabla \phi)^{2}$ changes sign at the horizon [7]. This provides us with a sensible local definition of an apparent horizon which will be useful in what follows.

\section{Quantum Corrections}

The most important result of the original paper of CGHS [4] is that the backreaction on the geometry due to Hawking radiation can be described by adding appropriate correction terms to the classical equations of motion. When there is a large number of matter fields in the theory there is a class of quantum corrections, associated with closed matter loops, which become important when $g^{2} N \sim 1$, long before other gravitational corrections kick in. At the one-loop level the matter conformal anomaly is the only term of this class and it will be responsible for the quantum back-reaction we will explore later on. When a black hole is formed the coupling at the horizon is $g^{2} \sim \frac{\lambda}{M}$ so our approximations remain valid so long as the mass remains large compared to $N \lambda$.

At one-loop order the anomaly contributes the well known non-local term

$$
-\frac{N}{96 \pi} \int d^{2} x \sqrt{-g} R \frac{1}{\nabla^{2}} R
$$

to the effective action [11]. In the conformal gauge (2.3) this term appears local, $-\frac{N}{12 \pi} \int d^{2} x \partial_{+} \rho \partial_{-} \rho$ and the one-loop corrected equations of motion, which we refer to as the CGHS-equations, can be arranged as follows,

$$
\begin{aligned}
\partial_{+} \partial_{-} f_{i} & =0 \\
\partial_{+} \partial_{-} \phi & =\left(1-\frac{N}{24} e^{2 \phi}\right) \partial_{+} \partial_{-} \rho \\
2\left(1-\frac{N}{12} e^{2 \phi}\right) \partial_{+} \partial_{-} \phi & =\left(1-\frac{N}{24} e^{2 \phi}\right)\left(4 \partial_{+} \phi \partial_{-} \phi+\lambda^{2} e^{2 \rho}\right) .
\end{aligned}
$$

$\star$ Following CGHS we assume that the Liouville cosmological constant term can be fine tuned to zero. 
The constraint equations also receive corrections,

$$
\begin{aligned}
& e^{-2 \phi}\left(2 \partial_{+}^{2} \phi-4 \partial_{+} \rho \partial_{+} \phi\right)=\frac{1}{2} \sum_{i=1}^{N} \partial_{+} f_{i} \partial_{+} f_{i}-\frac{N}{12}\left(\partial_{+} \rho \partial_{+} \rho-\partial_{+}^{2} \rho+t_{+}\left(x^{+}\right)\right) \\
& e^{-2 \phi}\left(2 \partial_{-}^{2} \phi-4 \partial_{-} \rho \partial_{-} \phi\right)=\frac{1}{2} \sum_{i=1}^{N} \partial_{-} f_{i} \partial_{-} f_{i}-\frac{N}{12}\left(\partial_{-} \rho \partial_{-} \rho-\partial_{-}^{2} \rho+t_{-}\left(x^{-}\right)\right)
\end{aligned}
$$

The functions $t_{ \pm}\left(x^{ \pm}\right)$are needed to satisfy asymptotic physical boundary conditions [4], so there remains a degree of non-locality in the conformal gauge. As expected the quantum corrections in these equations appear with factors of $N e^{2 \phi}$ compared to the classical terms.

For some purposes it can be useful to have a description of this system outside conformal gauge. In a more general gauge the anomaly induced term (3.1) can be made local by adding a new degree of freedom which is coupled to the scalar curvature,

$$
S_{Z}=\frac{1}{2 \pi} \int d^{2} x \sqrt{-g}\left[-\frac{1}{2}(\nabla Z)^{2}+\sqrt{\frac{N}{24}} Z R\right] .
$$

The non-local form (3.1) is recovered when $Z$ is integrated out of the path integral. The energy momentum tensor of the $Z$ field is

$$
T_{\mu \nu}=\frac{1}{2}\left[\nabla_{\mu} Z \nabla_{\nu} Z-\frac{1}{2} g_{\mu \nu}(\nabla Z)^{2}\right]+\sqrt{\frac{N}{24}}\left[\nabla_{\mu} \nabla_{\nu} Z-g_{\mu \nu} \nabla^{2} Z\right] .
$$

The conformal anomaly of the matter fields appears as a non-vanishing trace of the classical energy-momentum tensor of $Z$. This is analogous to what happens in bosonization of two-dimensional spinor electrodynamics where the axial anomaly of the fermions can be expressed classically in terms of the bosonizing field. We will develop this analogy further in section 5 when we discuss the fate of quantum information in this theory.

The classical equations (2.4) can be made linear by a combination of gauge choices and field redefinitions, which makes them relatively easy to solve. Unfortunately these tricks no longer work when the one-loop corrections have been added, and we now have coupled, non-linear, partial differential equations to deal with. It is easily checked that the linear dilaton vacuum (2.6) remains an exact solution. The geometry due to an incoming shock wave can no longer be found in closed form but is described by some non-trivial solution of the CGHS-equations which is matched continuously onto the vacuum across $x^{+}=x_{0}^{+}$. It approaches a classical black hole solution asymptotically far away, where the coupling is weak. 
In the remainder of this section we will use the equations of motion and constraints to explore qualitative features of such solutions, reinforcing and extending the observations of reference [7]. We will also present numerical calculations of two-dimensional black hole evolution which support our analytic results.

The first thing to note is that a curvature singularity forms on the infall trajectory at $e^{2 \phi}=\frac{12}{N}$. This was shown in [6] and [7] and we will not repeat the argument here. The singularity extends into a curve in the $x^{+}>x_{0}^{+}$region through which physical solutions cannot be meaningfully extended. Our approximations break down as the singularity is approached and it may or may not persist in the full quantum theory, but its presence at this level means that care must be taken in developing any systematic approximations. Perhaps one should view the $\frac{N}{12} e^{2 \phi}=1$ line as a natural boundary to spacetime in the quantum theory. We will at any rate focus on the evolution of massive black holes where the CGHS-equations are reliable so long as the mass remains large compared to $N \lambda$. Our results are for the most part independent of how the singularity issue is ultimately resolved. It is worth pointing out that removing the singularity by modifying the short distance physics will not automatically cure problems of information loss. The evaporation process would still be governed by the semi-classical equations for most of the black hole lifetime and there will be a limited amount of energy left to carry off the information contained in the original black hole (we are assuming for the moment that it does not get radiated away during the semi-classical evaporation). Either it would be contained in a light stable remnant or have to be emitted in very low energy radiation over a long time. In either case we would have to contend with a virtually infinite density of (meta-)stable states at the Planck scale in the quantum theory.

The singularity is cloaked by an apparent horizon which forms at $x^{-} \simeq-\frac{M}{\lambda^{3} x_{0}^{+}}$, for $M$ large, and then begins to recede as the black hole emits energy in Hawking radiation [7]. Later on we will show that the apparent horizon continues to recede as long as there is outgoing energy flux from the black hole. This intuitively obvious result provides a nice consistency check on the picture of black hole evaporation presented by the CGHS-equations.

The picture of black hole evaporation which emerges is qualitatively as follows. The curve of singularity at $\frac{N}{12} e^{2 \phi}=1$ forms inside the apparent horizon where $\nabla \phi$ is time-like and lines of constant $\phi$ are space-like. Thus it follows that the line of singularity is everywhere space-like unless it were to cross the apparent horizon. Such a crossing, however, can not happen since $\partial_{+} \phi$ equals zero on the apparent horizon but diverges on the singularity. Once the black hole has formed it emits Hawking radiation and the apparent horizon recedes. Since it cannot cross the singularity they must both approach light-like lines. We will present analytic and 
numerical evidence which strongly suggests that they in fact approach the same light-like line from opposite sides as in figure 2. The light-like asymptote is a global event horizon.

If all the mass of the black hole is radiated then one expects the geometry to approach the vacuum solution. Alternatively the black hole might approach a massive stable remnant whose properties would emerge at asymptotically late times. To investigate the asymptotic behavior of black hole solutions one can consider how coordinate invariants, such as the curvature scalar or $(\nabla \phi)^{2}$, evolve along contours of constant coupling strength. One can, for example, derive a simple relation between the rate of change of $R$ along a curve of constant $\phi$ and the flux of energy across the curve. For this purpose it is convenient to introduce local coordinates $u, v$ in the neighborhood of the contour, such that $u$ parametrizes the contour, $\phi\left(\tilde{x}^{+}(u), \tilde{x}^{-}(u)\right) \equiv \phi_{0}$, and $v$ parametrizes orthogonal curves. A good choice is to take $v$ as $\phi$ itself and normalize $u$ in such a way that the Jacobian determinant of the coordinate transformation from $x^{+}, x^{-}$to $u, v$ equals one. The matter energy, including Hawking radiation, in this coordinate system is $T^{u u}$ and the outgoing energy flux is $T_{u}^{v}$.

The curvature scalar in the conformal gauge (2.3) is given by

$$
R=8 e^{-2 \rho} \partial_{+} \partial_{-} \rho
$$

which can be reexpressed using the equations of motion (3.2) as

$$
R=\frac{4}{1-\frac{N}{12} e^{2 \phi}}\left(4 e^{-2 \rho} \partial_{+} \phi \partial_{-} \phi+\lambda^{2}\right) .
$$

We are interested in the rate of change of curvature along the $\phi=\phi_{0}$ contour,

$$
\begin{aligned}
\dot{R} & \left.\equiv \frac{d}{d u} R\right|_{\phi=\phi_{0}} \\
& =\frac{\partial \tilde{x}^{+}}{\partial u} \partial_{+} R+\frac{\partial \tilde{x}^{-}}{\partial u} \partial_{-} R \\
& =\partial_{-} \phi \partial_{+} R-\partial_{+} \phi \partial_{-} R .
\end{aligned}
$$

Inserting the expression (3.7) for the curvature gives

$$
\dot{R}=\frac{8 e^{-2 \rho}}{1-\frac{N}{12} e^{2 \phi_{0}}}\left[\left(\partial_{-} \phi\right)^{2}\left(2 \partial_{+}^{2} \phi-4 \partial_{+} \rho \partial_{+} \phi\right)-\left(\partial_{+} \phi\right)^{2}\left(2 \partial_{-}^{2} \phi-4 \partial_{-} \rho \partial_{-} \phi\right)\right] .
$$

The constraint equations (3.3) reveal that $e^{-2 \phi}\left(2 \partial_{ \pm}^{2} \phi-4 \partial_{ \pm} \rho \partial_{ \pm} \phi\right)$ is the gravitational response to the energy-momentum tensor of matter and Hawking radiation 
so we can write

$$
\dot{R}=\frac{8 e^{-2 \rho+2 \phi_{0}}}{1-\frac{N}{12} e^{2 \phi_{0}}}\left[\left(\partial_{-} \phi\right)^{2} T_{++}-\left(\partial_{+} \phi\right)^{2} T_{--}\right] .
$$

The final step is to observe that the expression in the square brackets is proportional to the energy flux across the contour and we obtain the desired result

$$
\dot{R}=-\frac{4 e^{2 \phi_{0}}}{1-\frac{N}{12} e^{2 \phi_{0}}} T_{u}^{v}
$$

This relationship can be checked by considering a contour in the classical region $N e^{2 \phi_{0}}<<1$. The value of the curvature of the classical solution (2.8) where the contour meets the infall line is $R=4 \lambda e^{2 \phi_{0}} M$. If the curvature on the contour goes to zero at late times then (3.11) implies that the integrated flux of Hawking radiation carries off an amount of energy equal to the original mass. In other words, the geometry approaches flat space if and only if the black hole radiates away all its energy.

We are now ready to show that the apparent horizon always recedes if there is any outgoing flux of energy from the black hole. Let the line of apparent horizon be parametrized as $x^{-}=\hat{x}^{-}\left(x^{+}\right)$. By definition $\partial_{+} \phi$ vanishes everywhere along this curve,

$$
0=\left.\frac{d}{d x^{+}} \partial_{+} \phi\right|_{x^{-}=\hat{x}^{-}}=\partial_{+}^{2} \phi+\left(\frac{d \hat{x}^{-}}{d x^{+}}\right) \partial_{+} \partial_{-} \phi .
$$

The constraints (3.3) relate the value of $\partial_{+}^{2} \phi$ on the apparent horizon to the outgoing energy flux at that point. Using this and the second equation of motion in (3.2) we can write the slope of the horizon curve in terms of the flux as follows,

$$
\frac{d \hat{x}^{-}}{d x^{+}}=\frac{e^{2 \phi}\left(1-\frac{N}{12} e^{2 \phi}\right)}{2 \lambda^{2}\left(1-\frac{N}{24} e^{2 \phi}\right)\left(\partial_{-} \phi\right)^{2}} T_{u}^{v} .
$$

The horizon recedes at a rate proportional to the outward flow of energy from the black hole (and advances when the black hole absorbs infalling matter).

On the apparent horizon the expression for the curvature (3.7) reduces to $R=\frac{4 \lambda^{2}}{\left(1-\frac{N}{12} e^{2 \phi}\right)}$ and it immediately follows that the horizon curvature increases as the black hole evaporates and the apparent horizon recedes to larger values of $e^{2 \phi}$.

The apparent horizon of a massive black hole forms in a region of weak coupling, $e^{2 \phi} \simeq \frac{\lambda}{M}$, and a good approximation to $\frac{d \hat{x}^{-}}{d x^{+}}$is obtained by inserting the classical 
solution (2.8) on the right hand side in (3.13). To leading order this gives [7]

$$
\frac{d \hat{x}^{-}}{d x^{+}}=\frac{N}{48 \lambda^{2} x_{0}^{+2}}
$$

which corresponds to a rate of energy loss of $\frac{N \lambda^{2}}{48}$ in agreement with the asymptotic flux of Hawking radiation found by CGHS [4]. So long as the remaining black hole mass is large compared to $N \lambda$ the rate of energy loss is slow compared to the mass and (3.14) (with $x_{0}^{+}$replaced by $x^{+}$) gives the instantaneous slope. Integration gives an 'adiabatic' approximation to the horizon curve,

$$
\hat{x}^{-}\left(x^{+}\right)=\hat{x}^{-}\left(x_{0}^{+}\right)+\frac{N}{48 \lambda^{2}}\left(\frac{1}{x_{0}^{+}}-\frac{1}{x^{+}}\right) .
$$

In the $x^{+} \rightarrow \infty$ limit this approaches a global horizon at $\hat{x}^{-}=\hat{x}_{0}^{-}+\frac{N}{48 \lambda^{2} x_{0}^{+}}$. For a very large initial mass this should be an excellent estimate because (3.14) is valid for most of the black hole lifetime. The approximation breaks down when the coupling strength becomes too large. Adiabatic arguments similar to those given above lead to a linearly growing coupling strength at the apparent horizon,

$$
e^{2 \phi}=\frac{\lambda x^{+}}{M x_{0}^{+}}
$$

which remains small for a long time if the original mass is large.

Note that in the $x^{ \pm}$coordinate system the total distance along $x^{-}$, which the apparent horizon recedes, is independent of the black hole mass. This means that the global event horizon extends far into the weak coupling region and at first sight it appears that some, if not almost all, of the information about the quantum state of the system will be lost behind it. However, as we shall soon see, the back-reaction is strong enough to convert all the energy of the infalling matter to outgoing Hawking radiation by the time the incoming shock wave reaches the global event horizon. We will address the question of information loss in section 5 and at that point it will be useful to have a clear notion of the total energy carried across light-like lines of constant $x^{-}$, such as the global horizon.

We will define the total energy on a given $x^{-}$line as

$$
M\left(x^{-}\right)=\lim _{x^{+} \rightarrow \infty} \frac{1}{4 \lambda}\left(1-\frac{N}{12} e^{2 \phi}\right)^{\frac{3}{2}} e^{-2 \phi} R .
$$

This definition gives the correct mass of classical black hole solutions. The factor of $\left(1-\frac{N}{12} e^{2 \phi}\right)^{\frac{3}{2}}$ does not affect this since it goes to 1 as $x^{+} \rightarrow \infty$ but it is convenient for 
what follows. For large negative $x^{-}$this expression for $M\left(x^{-}\right)$gives the standard initial total energy of the infalling matter in gravitational collapse. As $x^{-}$increases the remaining mass will be reduced by an amount equal to the energy that has been radiated out in Hawking radiation up to that point. Using the equations of motion and the constraints one obtains

$$
\frac{d M}{d x^{-}}=\frac{2}{\lambda} \partial_{+} \phi e^{-2 \rho} T_{--}^{m},
$$

where $T_{--}^{m}$ is the - component of the matter energy momentum tensor with the contribution from the anomaly included. The factors in front of $T_{--}^{m}$ reflect the fact that the $x^{+}, x^{-}$coordinate system is not asymptotically Minkowskian. Thus (3.17) appears to be a sensible definition of the mass and it will clearly go to zero at the global event horizon if all the energy of the black hole is radiated away. $M\left(x^{-}\right)$can be written as an integral as follows,

$$
M\left(x^{-}\right)=\frac{1}{4 \lambda} \int_{0}^{\infty} d x^{+} \frac{\partial}{\partial x^{+}}\left[\left(1-\frac{N}{12} e^{2 \phi}\right)^{\frac{3}{2}} e^{-2 \phi} R\right]
$$

and by using the equations of motion and the constraints this can be worked into,

$$
M\left(x^{-}\right)=\frac{1}{4 \lambda} \int_{0}^{\infty} d x^{+} \sqrt{1-\frac{N}{12} e^{2 \phi}} e^{-2 \rho} \partial_{-} \phi T_{++}^{m} .
$$

We will make use of this expression in section 5 .

We close this section by presenting some preliminary numerical results on black hole evolution in this $1+1$ dimensional model. We consider a black hole formed by an incoming shock wave. When viewed on lines of constant $x^{+}$the equations of motion (3.2) are ordinary differential equations for $\partial_{+} \phi$ and $\partial_{+} \rho$,

$$
\begin{aligned}
\partial_{-}\left(\partial_{+} \phi\right) & =\left(1-\frac{N}{24} e^{2 \phi}\right) \partial_{-}\left(\partial_{+} \rho\right) \\
2\left(1-\frac{N}{12} e^{2 \phi}\right) \partial_{-}\left(\partial_{+} \phi\right) & =\left(1-\frac{N}{24} e^{2 \phi}\right)\left(4 \partial_{-} \phi \partial_{+} \phi+\lambda^{2} e^{2 \rho}\right) .
\end{aligned}
$$

This was used in reference [7] to obtain exact information about the solution just above the infall line $x^{+}=x_{0}^{+}$and to establish the formation of the singularity at $\frac{N}{12} e^{2 \phi}=1$. The equations (3.21) can be solved exactly on the infall line because the functional coefficients in them are explicitly known there in terms of the linear dilaton background. But now the information about $\partial_{+} \phi$ and $\partial_{+} \rho$ on the infall 
line can be used to estimate the solution on the line $x^{+}=x_{0}^{+}+\epsilon^{+}$where $\epsilon^{+}$is some small step size. Then the ordinary differential equations (3.21) can be solved numerically for $\partial_{+} \phi$ and $\partial_{+} \rho$ on that line, making it possible to take another step in the $x^{+}$direction. Figure 3 shows typical results of evolving a gravitational collapse solution of the CGHS-equations in this way. The numerical integration cannot get through the singularity but since it lies on a space-like curve this does not affect the numerical solution in the physical region. The apparent horizon recedes as expected and it appears to approach the singularity asymptotically.

We have also located some contours of constant $\phi$ (which are not shown in figure 3) and evaluated the curvature scalar along them. The curvature does decrease along these contours as expected from (3.11). Numerical instability prevents us from following the contours very far into the $x^{+}$direction and we can not at present determine from our data whether $R$ goes to zero asymptotically. The hyperbolic nature of the CGHS-equations will make most numerical approaches unstable but one can certainly do better with a more sophisticated numerical method that the one we have employed here.

\section{Static Black Holes}

In this section we will discuss some properties of static black holes and speculate about their possible role as massive stable remnants of black hole evaporation. Some related issues are discussed in [12] We will present numerical evidence for the existence of static, non-radiating solutions of the CGHS-equations, which are candidates for stable $1+1$ dimensional remnants. ${ }^{\star}$ These solutions exist for any value of black hole mass and they have some interesting properties. In particular, the coupling is weak, $N g^{2}<<1$, everywhere in space if the mass is large, and thus it is possible that all further quantum corrections to these solutions remain small.

At large positive $x$ the static solutions rapidly tend to their classical counterparts of the same mass but there is no energy in Hawking radiation in this region. For a massive black hole, $M>>N \lambda$, the classical geometry is well approximated until one approaches the position of the classical horizon, but there, in a metrically small region, the metric and dilaton change radically. In particular the curvature first increases to a maximum value, which depends on the mass, and then rapidly decreases, changes sign and goes to minus infinity at a finite proper distance, as one advances in the minus $x$ direction. More surprisingly the dilaton reaches a

$\star$ These numerical solutions have also been obtained by J. Russo. While preparing this paper for publication we learned that B. Birnir, S. Giddings, J. Harvey and A. Strominger have found numerical static solutions to the CGHS-equations [13] and that they have also been studied by S. Hawking [14]. 
maximum value at the same place as the curvature and thereafter the coupling strength decreases monotonically to zero as one approaches the curvature singularity. The maximum coupling is always smaller than the coupling in the classical solution at the horizon, i.e. $g^{2}<\frac{\lambda}{M}$. This means that one might not expect the solution to receive any large corrections if $M>>N \lambda$.

As mentioned in the introduction, massive remnants of this sort offer the possibility of carrying off information about the quantum state of a black hole without leading to an infinite density of states at the Planck scale. The naked curvature singularity might pose problems of its own but these solutions are not ruled out on any a priori grounds. Later on we will consider the question of whether black hole evaporation can lead to one of these massive remnants as a final state. The answer turns out to be negative, at least in $1+1$ dimensions, for we are able to show that the gravitational collapse solutions of the CGHS-equations cannot approach massive static black holes asymptotically. This of course does not preclude the possibility of tunneling into one of these states at some stage in the evolution, but that process is presumably suppressed by large barrier factors for macroscopic black holes.

Before we can rule out the static solutions as the final states, we need to explore some of their properties. We begin with a brief digression on the quantum theory of a free field in Rindler space [15,16]. This simple system shares important features with black holes both in four and two spacetime dimensions.

The $|x|>|t|, x>0$ wedge of flat two-dimensional Minkowski space may be described by Rindler coordinates $R, \theta$ as follows,

$$
\begin{aligned}
t & =R \sinh \theta, \\
x & =R \cosh \theta,
\end{aligned}
$$

or $x^{ \pm}= \pm R e^{ \pm \theta}$. The Rindler metric is

$$
d s^{2}=-R^{2} d \theta^{2}+d R^{2}
$$

The Rindler Hamiltonian is conjugate to the time variable $\theta$ and is simply the generator of Lorentz boosts in Minkowski space. At $t=0$,

$$
H_{\mathrm{R}}=\int_{0}^{\infty} d x T^{00}(x) x
$$

The Rindler Hamiltonian only acts on a Hilbert space defined in terms of fields living on the positive $x$-axis and a second Hilbert space is needed in order to 
describe degrees of freedom on the negative $x$-axis. The Rindler Hamiltonian has a set of eigenvectors including a ground state. However, this ground state is not to be identified with the physical vacuum of Minkowski space, which has non-vanishing correlations between degrees of freedom on the positive and negative $x$-axis. These correlations are strongest at short distances and the Rindler ground state deviates violently from the physical vacuum very close to the origin. In fact, the expectation value of the Minkowski energy density diverges in the Rindler ground state at that point. Since the Minkowski vacuum is defined on the full $x$-axis it can not be a pure Rindler state but is rather a thermal density matrix, as is well known $[15,16]$.

An analogous situation arises when a black hole is described in Kruskal coordinates. In that case the "Rindler ground state" is singular at the horizon. It describes a matter field configuration with divergent energy density which is expected to react strongly back on the geometry at the horizon and therefore this state is usually not taken seriously. It nevertheless remains an open question whether there exists a corresponding zero temperature quantum state when gravitational corrections have been taken into account. It turns out that such a configuration can be found in our $1+1$ dimensional model, as we shall shortly see, and we expect one to exist in $3+1$ dimensions also. In fact, this is presumably the only static macroscopic field configuration in quantum gravity with a given mass. A finite temperature black hole in thermal equilibrium with its surroundings is not consistent because the thermal bath filling space would have infinite total energy. Of course, real black holes with finite Hawking temperature are not described by a static geometry but are formed in gravitational collapse. Hawking's original calculation showed that gravitational collapse results in a black hole with a non-singular horizon and outgoing radiation with a thermal spectrum, but the black hole is not in thermal equilibrium with the rest of space. Whilst it is well established that black holes are formed with non-vanishing Hawking temperature and emit radiation it is very unclear how they subsequently evolve. It is an intriguing possibility that the back-reaction of Hawking radiation on the geometry could cause the evaporating black hole to eventually settle into one of these massive zero temperature remnants, and we see no reason to reject this out of hand. We will, however, be able to show that this scenario is not realized in actual gravitational collapse, at least not in our $1+1$ dimensional toy model.

Let us first consider the energy momentum of quantum mechanical matter in a static black hole background ignoring back-reaction on the metric and dilaton. The static black hole solution (2.7) written in asymptotically Minkowskian coordinates $\tau, \zeta$ is 


$$
\begin{aligned}
& e^{-2 \rho}=1+\frac{M}{\lambda} e^{-2 \lambda \zeta}, \\
& e^{-2 \phi}=e^{2 \lambda \zeta}\left(1+\frac{M}{\lambda} e^{-2 \lambda \zeta}\right) .
\end{aligned}
$$

Far away from the black hole the metric is nearly flat and $\phi$ approaches the linear dilaton background. In Euclidean space this solution is easily seen to be a coordinate transformation of Witten's semi-infinite cigar [17], the cigar tip being at $\zeta \rightarrow-\infty$. The Hawking effect can be described in terms of the conformal anomaly [4,5], and it is convenient to express it in terms of the $Z$ field in (3.4). The curvature acts as a source term in the $Z$ equation of motion,

$$
\nabla^{2} Z=-\sqrt{\frac{N}{24}} R .
$$

The cigar is flat far away from the horizon and one finds a static $Z$ field linear in the spatial coordinate,

$$
Z \simeq \sqrt{\frac{N}{6}} \zeta
$$

Inserting this into (3.5) gives a constant energy density, $\frac{N}{24}$, of Hawking radiation. The coefficient $\sqrt{\frac{N}{6}}$ only involves the integrated curvature, or Euler number, of the cigar end, making it clear that Hawking temperature is independent of mass in $1+1$ dimensions. There also exists a solution for $Z$ which has vanishing energy density far away but that requires a point source of $Z$ at the cigar tip and this causes the energy density to diverge at the horizon. This singular configuration is the zero temperature "Rindler ground state" *

Neither the thermal black hole nor the zero temperature one remain consistent solutions when back-reaction is taken into account. As we mentioned before a thermal static configuration can not be consistent for it would have infinite total energy. On the other hand it is reasonable to look for a zero temperature static solution of the CGHS-equations with some finite mass (or ADM-energy).

Static fields $\phi, \rho$ only depend on the spatial variable $\zeta$ and satisfy the following set of ordinary differential equations in conformal gauge,

$$
\begin{aligned}
\phi^{\prime \prime} & =\left(1-\frac{N}{24} e^{2 \phi}\right) \rho^{\prime \prime} \\
\left(1-\frac{N}{12} e^{2 \phi}\right) \phi^{\prime \prime} & =2\left(1-\frac{N}{24} e^{2 \phi}\right)\left(\phi^{\prime 2}+\lambda^{2} e^{2 \rho}\right),
\end{aligned}
$$

$\star$ Once we are willing to entertain such a singularity the temperature in the asymptotic region can of course have any value. 
along with one constraint which can be written,

$$
\phi^{\prime 2}-\rho^{\prime} \phi^{\prime}+\frac{N}{48} \rho^{\prime 2}-\lambda^{2} e^{2 \rho}=0 .
$$

The classical static black hole (4.4) is a solution of the equations without the $N e^{2 \phi}$ terms. Far away from the black hole the correction terms tend to zero and a given solution of the full equations matches onto a classical one with the same ADMenergy. Unfortunately we have not been able to find the general solution to the full set of non-linear equations (4.7) in closed form, but they can easily be handled numerically. In figures 5 to 6 we display some results of numerical integration. We plot both the value of $e^{2 \phi}$ and the scalar curvature as a function of spatial position for af number of solutions of (4.7) with different black hole mass. Figure 4 graphs the coupling strength and curvature vs. position in the classical solution (4.4) for comparison.

The figures clearly demonstrate the behavior alluded to at the beginning of this section. In particular, we see that the geometry and dilaton approach a classical black hole solution at large distances but before the classical horizon is reached the curvature reaches a maximum and the decreases to $-\infty$. Simultaneously the coupling strength $e^{2 \phi}$ reaches a maximum and then tends to zero as the curvature tends to $-\infty$. The maximum coupling is always less than the critical value, $\frac{N}{12} e^{2 \phi_{\max }}<1$, but approaches this value as $M \rightarrow 0$. The curvature reaches a maximum positive value before it dips down to $-\infty$. As $M \rightarrow 0$ this maximum positive curvature diverges but at the same time the size of the region which deviates from the classical solution goes to zero.

The solutions found above are the only static solutions of the CGHS-equations. As expected, no solutions exist which describe a static black hole in thermal equilibrium with its surroundings.

A natural question to ask is whether a black hole formed in a physical process such as collapse can evolve into a massive zero temperature object described by these static solutions. We will now show that the CGHS-equations do not have solutions in which this occurs. That may not prevent quantum mechanical tunneling into a massive zero temperature configuration at some stage in the evolution. However, we expect that to be heavily suppressed at least until the black hole has lost almost all its mass.

We begin by noting that in the gravitational collapse of a shock wave the dilaton field increases monotonically on the infall line $x^{+}=x_{0}^{+}$, until it hits the singularity at $\frac{N}{12} e^{2 \phi}=1$. We will prove that this continues to hold on every line of constant $x^{+}$, i.e. that it is not possible for the dilaton field to develop a local maximum 
at $\frac{N}{12} e^{2 \phi}<1$ as is found in all $M>0$ static solutions. We know that on each such line $e^{2 \phi}$ varies continuously from zero, as $x^{-} \rightarrow-\infty$, to $\frac{12}{N}$ at the singularity. (Recall that the singularity is a space-like curve which asymptotically approaches the light-like line, $x^{-}=H$, as shown in figure 2.) Thus if a local maximum occurs it must be accompanied by a local minimum closer to the singularity, as illustrated in figure 7 . Call the points where the extrema occur $x_{1}^{-}\left(x^{+}\right)$and $x_{2}^{-}\left(x^{+}\right)$. At these points $\partial_{-} \phi=0$ and from the second equation of motion in (3.2) it is seen that $\partial_{+} \partial_{-} \phi>0$ there. It follows that the two curves $x_{1}^{-}\left(x^{+}\right)$and $x_{2}^{-}\left(x^{+}\right)$must approach one another as $x^{+}$increases. However, since the $\phi$ is monotonic on the infall line there must be a smallest value of $x^{+}$where the extrema first occur and at which $x_{1}^{-}=x_{2}^{-}$. Since the equations of motion prevent $x_{1}^{-}$and $x_{2}^{-}$from separating it follows that $\phi$ must remain monotonic.

The local maxima of $\phi$ as a function of the spatial variable $\zeta$ are also local maxima when viewed along constant $x^{+}$lines. Therefore the CGHS-equations do not permit infalling matter to evolve to a massive remnant. The only static solution which has monotonic $\phi$ is the $M=0$ solution. It seems highly plausible that the asymptotic endpoint of $1+1$ dimensional black hole evolution is the $M=0$ state. Late in the evolution the system is expected to become highly quantum mechanical and the above argument does not rule out the possibility of forming light stable remnants.

The approach to the vacuum state, as described by the CGHS-equations, is subtle and non-uniform. We believe that along any line of constant $\phi$ in the physical region, $\frac{N}{12} e^{2 \phi}<1$, the evaporating solution will eventually tend to the vacuum configuration. However, this cannot occur until the apparent horizon has receded beyond that value of $\phi$. As $x^{+}$increases there will always be a small but diminishing region which remains inside the apparent horizon. In this region the solution is qualitatively different from the vacuum, for example constant $\phi$ contours are space-like there, but any given $\phi$ contour will eventually emerge outside the horizon and become vacuum-like. It is unclear how much physical meaning should be attached to the Liouville region, $\frac{N}{12} e^{2 \phi}>1$. For our purposes the singularity can be taken as a boundary of spacetime. 


\section{The Fate of Quantum Information}

If stable remnants do not store the information that falls into a black hole then the only way to have a unitary quantum theory is to find a complete correlation between the state of the infalling matter which formed the black hole and the final state of the Hawking radiation. The fact that the final radiation is approximately thermal is in no way inconsistent with this. However, it is important to exhibit a mechanism for transferring the infalling information to the outgoing particles. Consider figure 8 which depicts an infalling system with some complexity forming a black hole and subsequently radiating away its energy. In order that the Hawking radiation carry away all the information it is necessary that the infalling matter has had all its information eliminated before it crosses the global event horizon at $x^{-}=H$. If the infalling matter has any memory of the initial state beyond this point then the Hawking radiation cannot be in a pure state since it would still be correlated to the infalling matter which has passed inside the event horizon. It is not sufficient for the infalling matter to imprint its state on the outgoing radiation like DNA imprinting itself on RNA. The original information in the "DNA" must also be destroyed if the "RNA" is to be in a pure state. At first sight this seems obviously impossible because the $f$-waves satisfy free field equations of motion and any structure that they have is maintained all along the infall line. In fact a free massless left-moving $f$ field has an infinite number of conserved quantities which characterize the exact shape of the wave.

On the other hand, since all the energy of the system is emitted as Hawking radiation the total energy of the infalling matter must be zero when it arrives at $x^{-}=H$. One might wonder whether all the apparent information carried by the $f$ fields is real. At the moment we have no definitive answer to this important question but we can exhibit a similar system in which the apparent information in an infalling free field is illusory and in which all real information is radiated in an analog of Hawking radiation.

Consider the $1+1$ dimensional Schwinger model ${ }^{\star}$ with a coupling constant which depends on position like

$$
g^{2}=e^{2 x}
$$

where $x$ is the spatial coordinate. This can be thought of as electrodynamics in the linear dilaton vacuum. The Lagrangian is

$$
L=-\frac{1}{4 g^{2}(x)} F_{\mu \nu} F^{\mu \nu}+i \bar{\psi} \gamma^{\mu}\left(\partial_{\mu}-i A_{\mu}\right) \psi
$$

^ 1+1 dimensional electrodynamics has been considered in a different context of black hole physics in reference [18]. 
The analog for vector fields of the conformal gauge is the light-cone gauge $A_{-}=0$. In this gauge the Lagrangian becomes

$$
L=-\frac{1}{g^{2}(x)}\left(\partial_{-} A_{+}\right)^{2}+i \bar{\psi}_{L} \frac{\partial}{\partial x^{-}} \psi_{L}+i \bar{\psi}_{R}\left(\frac{\partial}{\partial x^{-}}-i A_{+}\right) \psi_{R}
$$

The field $\psi_{L}$ describing "infalling" fermions appears to be a free field. A message could be encoded in sequence of particles and antiparticles of this type. Naively, any such information in $\psi_{L}$ is conserved and penetrates indefinitely into the strong coupling region. However, this is incorrect. To see this let us integrate out the $\psi$ field. As is well known this contributes a one-loop correction to the effective action of $F_{\mu \nu}$, which is given by,

$$
\frac{1}{8 \pi} \int d^{x} \epsilon^{\mu \nu} F_{\mu \nu} \frac{1}{\nabla^{2}} \epsilon^{\lambda \sigma} F_{\lambda \sigma}
$$

Note the similarity with the Liouville action (3.1) obtained by integrating out the matter fields $f_{i}$. This action is non-local but we can introduce a $Z$ field as before. The effective Lagrangian then becomes

$$
L=\frac{1}{2} \partial_{\mu} Z \partial^{\mu} Z+\frac{1}{\sqrt{4 \pi}} \epsilon^{\mu \nu} F_{\mu \nu} Z-\frac{1}{4 g^{2}(x)} F_{\mu \nu} F^{\mu \nu}
$$

Now integrate the gauge field $A_{\mu}$ to obtain

$$
L_{\mathrm{Z}}=\frac{1}{2} \partial_{\mu} Z \partial^{\mu} Z+\frac{g^{2}(x)}{2 \pi} Z^{2}
$$

This is the bosonized form of the Schwinger model in which the axial anomaly appears at the classical level. In terms of $Z$ the charge current is given by $\epsilon^{\mu \nu} \partial_{\nu} Z$.

It is evident from (5.6) that information cannot penetrate indefinitely into the strong coupling region $x \rightarrow-\infty$. The effective mass of the bosonizing field $Z$ grows to infinity with the coupling so that any incoming $Z$ wave of finite energy is completely reflected. This simply indicates the fact that electric current cannot flow into the infinitely strongly coupled region.

The possibility of information penetrating the strong coupling region depends in detail on the model. For example if more than one fermion species carries charge then bosonization reveals one "massive" boson $Z$ with mass $\sim g^{2}(x)$ and a set of massless bosons which can carry information toward $x \rightarrow-\infty$. On the other hand if all currents are independently gauged then all the boson fields are reflected. 
It is not clear whether or not a theory can be constructed in which information is always drained out of the $f$ fields before they slip behind the global event horizon. It may be that an arbitrary theory, for example one which has global symmetries, cannot satisfy this. Perhaps it is only in some theories, or even only one, that black hole evolution can be made consistent with conservation of information.

A possible candidate for such a theory in $1+1$ dimensions is obtained if we completely replace the $f$ fields in the CGHS-model by the field $Z$. This way the matter carries no information which is uncoupled to gravity. The question is whether in such a theory a configuration on the global event horizon which carries zero total energy must also carry no information. For example if $T_{++}^{m}$ in our expression for the energy (3.20) were a positive quantity as it would be in a classical theory then vanishing energy would require the $Z$ field be trivial on the event horizon and therefore carry no information. Unfortunately energy density does not have to be positive in quantum theory.

To begin to address this question consider an incoming configuration described asymptotically at large negative $x^{-}$by some left-moving positive energy density $T_{++}\left(x^{+}\right)$. It is always possible to find a $Z$ field which carries the same energy distribution,

$$
T_{++}\left(x^{+}\right)=\frac{1}{2} \nabla_{+} Z \nabla_{+} Z+\sqrt{\frac{N}{24}} \nabla_{+}^{2} Z .
$$

Along a given line of fixed $x^{-}$one can find a coordinate transformation $x^{+} \rightarrow$ $y^{+}\left(x^{+}\right)$which renders the metric flat along that line. The new coordinates are still conformal and (5.7) can be written as

$$
T_{++}\left(x^{+}\right)=\frac{24}{N} e^{-\sqrt{\frac{N}{24}} Z}\left(\frac{\partial}{\partial y^{+}}\right)^{2} e^{\sqrt{\frac{N}{24}} Z}
$$

along the line in question. If we write $\psi=e^{\sqrt{\frac{N}{24}} Z}$ then (5.8) takes a familiar form. It is the time independent Schrödinger equation for a zero energy state in a potential $V\left(x^{+}\right)=\frac{N}{24} T_{++}\left(x^{+}\right)$. We can impose a boundary condition that $Z=0$ or $\psi=1$ in the linear dilaton vacuum at $x^{+}<x_{0}^{+}$. If we assume that $T_{++}$vanishes for large enough $x^{+}$then the solution of the Schrödinger equation will be linear in that region. If the sign of the linear term is negative then $\psi$ will have to go through zero at some point, and $Z$ becomes singular and ill-defined there. The sign depends on the potential $V$. For example, if $\int V\left(x^{+}\right) d x^{+} \leq 0$ a zero energy solution of the Schrödinger equation always has a node somewhere. This means that an incoming asymptotic configuration with negative total energy would necessarily be singular and the only non-singular zero energy state has $T_{++}=0$. 
In the case at hand it is not the incoming total energy which vanishes but rather the energy on the global event horizon. This condition does not appear to be strong enough to force $Z$ to be vacuum-like along the entire global horizon. To see that introduce a $y^{+}$coordinate system with flat metric on the global event horizon, $x^{-}=H$. The expression for the (vanishing) total energy (3.20) becomes

$$
\int_{0}^{\infty} d x^{+} \partial_{-} \phi \sqrt{1-\frac{N}{12} e^{2 \phi}}\left[\psi^{-1}\left(\frac{\partial}{\partial x^{+}}\right)^{2} \psi\right]=0 .
$$

In general the weight factor $\partial_{-} \phi \sqrt{1-\frac{N}{12} e^{2 \phi}}$ is not constant and that prevents us from concluding that $Z$ must be trivial. In fact the weight factor increases with increasing $x^{+}$and this might allow $T_{++}$to be positive on and near the infall line but to take a smaller compensating negative value at larger $x^{+}$. Unless the backreaction on the geometry provides more stringent constraints on $T_{++}$on the global horizon then even in this "minimal" theory all information will not be drained out of the incoming $Z$ field.

Acknowledgement: We thank J. Russo for numerous discussion and collaboration. We have also benefitted from discussions with M. Alford, B. Birnir, C. Callan, S. Giddings, G. Horowitz, J. Preskill, M. Srednicki, and A. Strominger

\section{REFERENCES}

1. S. W. Hawking, Comm .Math. Phys. 43 (1975), 199.

2. S. W. Hawking, Phys. Rev. D14 (1976), 2460.

3. G. 't Hooft, Nucl. Phys. B335 (1990), 138, and references therein.

4. C. G. Callan, S. B. Giddings, J. A. Harvey and A. Strominger, Evanescent black holes, preprint UCSB-TH-91-54, EFI-91-67, PUPT-1294, November 1991.

5. S. M. Christensen and S. A. Fulling, Phys. Rev. D15 (1977), 2088.

6. T. Banks, A. Dabholkar, M. R. Douglas and M. O'Loughlin, Are Horned Particles the Climax of Hawking Evaporation?, Rutgers University preprint, RU-91-54, January 1992.

7. J. G. Russo, L. Susskind and L. Thorlacius, Black Hole Evaporation in $1+1$ Dimensions, Stanford University preprint, SU-ITP-92-4, January 1992.

8. Y. Aharonov, A. Casher and S. Nussinov, Phys. Lett. 191B (1987), 51.

9. G. W. Gibbons and K. Maeda, Nucl. Phys. B298 (1988), 741. 
10. D. Garfinkle, G. Horowitz and A. Strominger, Phys Rev D43 (1991), 3140.

11. A. M. Polyakov, Phys. Lett. 163B (1981), 207.

12. S. B. Giddings, Black Holes and Dark Remnants, UCSB preprint, in preparation.

13. B. Birnir, S. B. Giddings, J. A. Harvey and A. Strominger, Quantum Black Holes, preprint UCSB-TH-92-08, EFI-92-16, March 1992.

14. S. W. Hawking, Evaporation of Two Dimensional Black Holes, Caltech preprint, CALT-68-\#1, March 1992.

15. W. G. Unruh, Phys. Rev D14 (1976), 870.

16. W. G. Unruh and R. M. Wald, Phys. Rev. D29 (1982), 1047.

17. E. Witten, Phys. Rev. D44 (1991), 314.

18. M. Alford and A. Strominger, S-Wave Scattering of Charged Fermions by a Black Hole, preprint NSF-ITP-92-13, February 1992. 